\title{
ANTIBIOTIC SUSCEPTIBILITY PATTERN OF Shigella spp. ISOLATED FROM PATIENTS SUSPECTED OF ACUTE GASTROENTERITIS
}

\author{
Bishal Basnet $^{1}$, Dhirendra Niroula ${ }^{1, *}$, Jyoti Acharya ${ }^{2}$, Shaila Basnyat ${ }^{1}$ \\ ${ }^{1}$ Central Department of Microbiology, Tribhuvan University, Kirtipur, Kathmandu, Nepal \\ ${ }^{2}$ Sukraraj Tropical Hospital and Research Center, Teku, Kathmandu, Nepal \\ *Corresponding author: dhiren.niroula@gmail.com
}

(Received: April 02, 2021; Revised: September 20, 2021; Accepted: September 22, 2021)

\begin{abstract}
Shigellosis, an intestinal infection caused by Shigella species, is manifested by bloody diarrhea. Due to the surge in multidrug-resistant (MDR) Shigella species, the control of shigellosis has been a big challenge. This study aims to determine the prevalence and assess the antibiotic susceptibility pattern of Shigella species. During our study period of five months from April 2014 to August 2014 at Sukraraj Tropical and Infectious Disease Hospital, Teku, Kathmandu, a total of 653 stool samples were collected from the patients suspected of acute gastroenteritis. The standard microbiological procedure was followed for the isolation and identification of Shigella species. Assessment of antibiotic susceptibility pattern of the Shigella species was done by Kirby-Bauer disk diffusion method following CLSI guidelines. The study found 25(3.82\%) cases were Shigella positive. Among them, 18(72\%) were S. flexneri, 6(24\%) were S. dysenteriae, and $1(4 \%)$ was $S$. sonnei. The patients in the age group 16-45 years were highly susceptible to infection as the higher proportion 16(64\%) of Shigella species were isolated from this age group ( $>>0.05)$. Shigella species were found to be highly susceptible to Cefotaxime (100\%), a third-generation cephalosporin. Nalidixic acid, on the other hand, was the least effective antibiotic as $20(80 \%)$ of the Shigella isolates were resistant, followed by Ampicillin 18(72\%), Cotrimoxazole 13(52\%), and Ciprofloxacin 9(36\%). A higher proportion of $[10(40 \%)]$ of our study isolates were MDR. Our results show that Nalidixic acid, Ampicillin, Cotrimoxazole, Ciprofloxacin, and Ofloxacin cannot be used as empirical therapy for the treatment of Shigella infection as Shigella species were highly resistant to these antibiotics. So, for the MDR Shigella infection, we suggest third-generation cephalosporin as an option.
\end{abstract}

Keywords: Antibiotic susceptibility, empirical therapy, MDR, Shigella species

\section{INTRODUCTION}

Shigella, a food-borne pathogen, is responsible for acute invasive gastroenteritis and traveler's diarrhea (von Seidlein et al., 2006). It is a major global health problem as it is a prime cause of diarrhea, with 212,000 deaths every year worldwide (Arnold, 2021). It is a major cause of mortality and morbidity in children under the age of five in developing nations (Kotloff et al., 2018). It is manifested by bloody diarrhea and is caused by Shigella flexneri, S. sonnei, S. boydii, or $S$. dysenteriae (Heymann, 2008). S. flexneri is the major cause of diarrhea in the least developed and developing countries, whereas $S$. sonnei is the major species isolated in the developed nations (Kotloff et al., 2018; Bardsley et al., 2020). The organism is usually transmitted by the feco-oral route when contaminated food or water is ingested. The definitive diagnosis of Shigella infection is done by isolating the organism from the stool and serotyping the isolate (WHO, 2005).

The severity of shigellosis and potential complications can be avoided by the proper use of antimicrobials (Lima et al., 1995); however, antibiotic resistance to Shigella species and serotypes has emerged throughout the world (Taneja et al., 2012). Previously effective firstline drugs such as Sulphonamide-trimethoprim, Ampicillin, and Nalidixic acid are almost ineffective against the prevalent Shigella strains (Kotloff et al., 2018; Puzari et al., 2018). With the recent rise in fluoroquinolone-resistant strains, the choice of drugs for effective antimicrobial therapy has vastly reduced (Kotloff et al., 2018; Puzari et al., 2018). As an alternative antimicrobial therapy, WHO has recommended three antibiotics, namely Pivmecillinam, Ceftriaxone, and Azithromycin (WHO, 2005). However, resistance to these WHO-recommended drugs has been observed as suggested by the findings of various studies.

In Nepal, S. flexneri is the predominant species, followed by S. dysenteriae (Wilson et al., 2006; Khan et al., 2013). Studies conducted in Nepal have found that most patients who are vulnerable to infection and develop infection are under the age of ten (Bhattacharya et al., 2005; Khan et al., 2013). Nepal has experienced Shigella 
Antibiotic Susceptibility Pattern of Shigella spp. Isolated from Patients ...

species resistance to multiple drugs over the past two decades. There are a few reasons for the emergence of antibiotic-resistant strains in Nepal, such as easy access to antibiotics in the pharmacies without a doctor's prescription, poor antibiotic policies, including poor laboratory diagnosis and antibiotic susceptibility tests (Dhital et al., 2017). Therefore, this study aims to provide more insight into the prevalence and the antibiotic susceptibility pattern of Shigella species isolated in a tertiary care hospital in Kathmandu.

\section{MATERIALS AND METHODS}

This cross-sectional study was done in Sukraraj Tropical and Infectious Disease Hospital, Teku, Kathmandu, over five months from April to August 2014.

\section{Patients and sample collection}

A total of 653 stool samples were collected from the patients complaining of acute gastrointestinal disorder, i.e., patients with fever, fatigue, frequent defecation of painful stools containing blood and mucus defined by physicians as a probable case of bacillary dysentery. Therefore, the stool samples containing blood and mucus from patients with no antibiotics taken within three days were included as study samples, whereas those with firm consistency without blood and mucus were excluded from the study. One gram of stool samples was collected in clean screw-capped plastic containers. The samples were labeled with the patient's name, age, sex, and address. If there was a possible delay in the processing of the sample, it was stored in the refrigerator at $2-8{ }^{\circ} \mathrm{C}$. The stool samples were further processed in the microbiology laboratory following standard operating procedures (McCartney et al., 1989).

\section{Bacteriological analysis}

A loopful of stool sample from each container was then inoculated in the Xylose lysine deoxycholate agar (XLD) and Mac-Conkey agar (MAC) plates and incubated at $37^{\circ} \mathrm{C}$ for 24 hours for the isolation of Shigella species. Simultaneously, enrichment was done in Selenite-F broth and incubated at $37^{\circ} \mathrm{C}$ for 24 hours. Subculture was then done in XLD agar and MAC and further incubated at $37^{\circ} \mathrm{C}$ for 24 hours. The colony morphology resembling Shigella species was identified microscopically and biochemically. The non-lactose fermenting (NLF) colonies from both XLD and MAC were identified by the IMViC test, Urease test, Nitrate reduction test, and Triple sugar iron (TSI) test.

\section{Serological identification}

Differentiation of Shigella species was done by slide agglutination test using polyvalent antisera (Denka Seiken Co. Ltd. Japan). On the clean glass slide, milky suspension of the organism was made with saline. Shigella polyvalent antiserum was added starting from A to D polyvalent. The mixture was tilted to and fro for 30-60 seconds. While viewing under a good light against a dark background, distinct clumping within 60 seconds was a positive result. The suspension alone, on the other hand, as control was tested to exclude auto-agglutination. The appearance of agglutination (distinct clumping) by Group A polyvalent, Group B polyvalent, Group C polyvalent, and Group D polyvalent in kit were identified as $S$. dysenteriae, S. flexneri, S. boydii, and S. sonnei respectively.

\section{Antibiotic susceptibility test}

The antibiotic susceptibility test was done by the KirbyBauer disk diffusion method in Muller-Hinton Agar (MA) following 2014 CLSI guidelines (CLSI, 2014). The antibiotic susceptibility pattern of six antibiotics, namely Ampicillin (Amp, $10 \mu \mathrm{g}$ ), Cefotaxime (Ctx, $30 \mu \mathrm{g}$ ), Cotrimoxazole (TMP-SMX, $25 \mu \mathrm{g}$ ), Ciprofloxacin (Cip, $5 \mu \mathrm{g})$, Ofloxacin (Ofx, $15 \mu \mathrm{g}$ ), and Nalidixic acid (Nal, 30 $\mu \mathrm{g})$ (Hi Media Laboratory Ltd. Mumbai, India) was assessed. The zone of inhibition of each antibiotic was measured, and they were interpreted as sensitive, intermediate sensitive, and resistant (CLSI, 2014). For the quality control purpose, strict aseptic conditions and techniques were followed according to the National Committee for Clinical Laboratory Standard (NCCLS) recommendation. Positive and negative control was always used during each testing with the standard organism. For the antibiotic susceptibility test, standard organism ATCC 25922 Escherichia coli was used for the quality control.

\section{Statistical analysis}

Data from the laboratory findings was analyzed using Microsoft Excel 365. The t-test was performed to compare the statistical significance. The relationship with a p-value greater than 0.05 was indicated as insignificant.

\section{Ethics clearance}

The written informed consent was taken prior to the study from the patients involved in the study. Our study was accepted by the Institutional Review Committee (IRC) at Sukraraj Tropical and Infectious Disease Hospital, Teku, Kathmandu.

\section{RESULTS}

Out of a total of 653 shigellosis suspected cases, $25(3.82 \%)$ were confirmed as positive cases (Fig. 1, Table 1). Among 25 positive shigellosis cases, S. flexneri, $S$. dysenteriae, and $S$. sonnei were found to be $18(72 \%)$, $6(24 \%)$, and 1(4\%), respectively (Fig. 1, Table 2). Shigellosis positive female patients were found to be comparatively higher than male patients (female$14(56 \%)$, male- $11(44 \%)$ ) (Table 1). The highest number of Shigella species were recovered from the age group 1645 years $16(64 \%)$ followed by age group $>45$ years $7(28 \%)$, whereas $2(8 \%)$ of the Shigella species was isolated from the age group $<16$ years (Table 2). However, there was no significant association between age and the number of species. 
The antibiotic susceptibility pattern of Shigella species suggests that Cefotaxime, the third-generation cephalosporin, was found to be the most effective (100\% sensitive) against all the isolated Shigella species (Table 3). Among the tested antibiotics, Nalidixic acid was the least effective as $20(80 \%)$ of Shigella species were resistant to it (Table 3). The second least effective antibiotic was
Ampicillin, as $18(72 \%)$ of Shigella species were resistant to it (Table 3). This was followed by Cotrimoxazole $13(52 \%)$ and Ciprofloxacin 9(36\%), respectively. Similarly, out of a total of 25 isolates, 10 isolates were found to be MDR, representing $40 \%$ of the total isolates (Fig. 2).
Culture Positivity

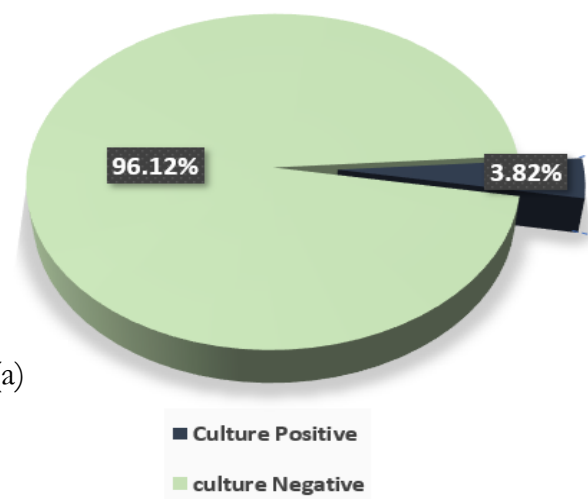

Shigella species

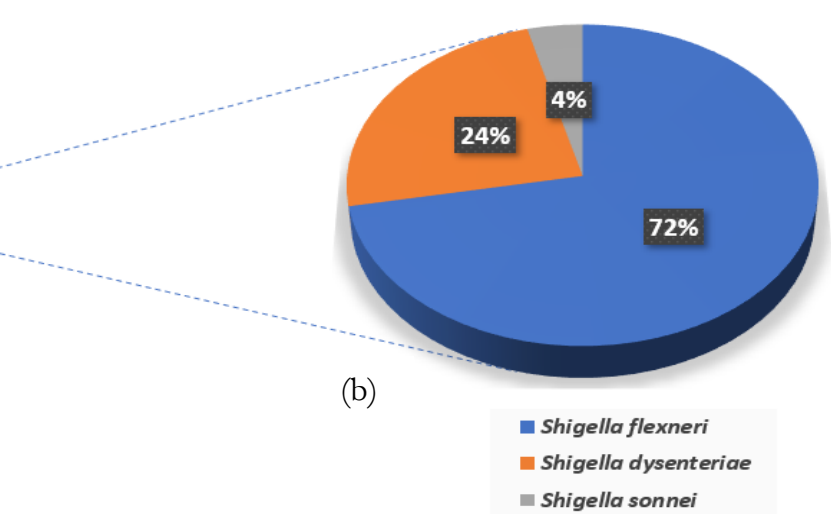

Figure 1. (a) The pie-chart represents the fraction of culture-positive isolates, (b) the pie-chart represents the proportion of Shigella species within the culture-positive isolates.

Table 1. Gender wise distribution of Shigella positive and negative patients

\begin{tabular}{|c|c|c|c|}
\hline \multirow[t]{2}{*}{ Sex } & \multicolumn{2}{|c|}{ Culture } & \multirow[t]{2}{*}{ Total } \\
\hline & $\begin{array}{l}\text { Positive } \\
(\%)\end{array}$ & $\begin{array}{c}\text { Negative } \\
(\%)\end{array}$ & \\
\hline Male & $11(44)$ & $287(45.7)$ & $298(45.6)$ \\
\hline Female & $14(56)$ & $341(54.3)$ & $355(54.4)$ \\
\hline Total & $25(3.82)$ & $628(96.12)$ & $653(100)$ \\
\hline
\end{tabular}

*The figures in the parentheses indicate the percentage

Table 2. Number of Shigella species isolated in different age groups

\begin{tabular}{lcccc}
\hline Age groups (years) & \multicolumn{3}{c}{ Shigella species } & \multirow{2}{*}{ Total (\%) } \\
\cline { 2 - 5 } & S. flexneri & S. dysenteriae & S. sonnei & \\
\hline$<16$ & 2 & 0 & 0 & $2(8)$ \\
$16-45$ & 10 & 5 & 1 & $16(64)$ \\
$>45$ & 6 & 1 & 0 & $7(28)$ \\
\hline Total (\%) & $18(72)$ & $6(24)$ & $1(4)$ & $25(100)$ \\
\hline
\end{tabular}

*The figures in the parentheses indicate the percentage

Table 3. The antibiotic susceptibility pattern of $S$. flexneri, $S$. dysenteriae, and $S$. sonnei

\begin{tabular}{|c|c|c|c|c|c|c|c|c|c|c|}
\hline \multirow{2}{*}{$\begin{array}{l}\text { Antibio } \\
\text { tics }\end{array}$} & \multicolumn{3}{|c|}{ S. flexneri $(\mathrm{n}=18)$} & \multicolumn{3}{|c|}{ S. dysenteriae $(\mathrm{n}=6)$} & \multicolumn{3}{|c|}{ S. sonnei $(\mathrm{n}=1)$} & \multirow{2}{*}{$\begin{array}{l}\text { Resistan } \\
\text { ce total } \\
(\%)\end{array}$} \\
\hline & $\mathrm{S}$ & I & $\mathrm{R}$ & S & I & $\mathrm{R}$ & S & I & $\mathrm{R}$ & \\
\hline Amp & $1(5.6)$ & $3(16.6)$ & $14(77.8)$ & $1(16.7)$ & $2(33.3)$ & $3(50)$ & $0(0)$ & $0(0)$ & $1(100)$ & $18(72)$ \\
\hline $\begin{array}{l}\text { TMP } / \mathrm{S} \\
\text { MX }\end{array}$ & $4(22.2)$ & $4(22.2)$ & 10(55.6) & $3(50)$ & $0(0)$ & $3(50)$ & 1(100) & $0(0)$ & $0(0)$ & $13(52)$ \\
\hline Cip & $4(22.2)$ & $6(33.3)$ & $8(44.5)$ & $5(83.3)$ & $1(16.7)$ & $0(0)$ & $0(0)$ & $0(0)$ & $1(100)$ & $9(36)$ \\
\hline Ofx & $6(33.3)$ & $7(38.9)$ & $5(27.8)$ & $4(66.7)$ & $2(33.3)$ & $0(0)$ & $0(0)$ & $0(0)$ & $1(100)$ & $6(24)$ \\
\hline Nal & $1(5.5)$ & $2(11.1)$ & 15(83.4) & $1(16.6)$ & $1(16.6)$ & $4(66.8)$ & $0(0)$ & $0(0)$ & $1(100)$ & $20(80)$ \\
\hline Ctx & $18(100)$ & $0(0)$ & $0(0)$ & $6(100)$ & $0(0)$ & $0(0)$ & $1(100)$ & $0(0)$ & $0(0)$ & $0(0)$ \\
\hline
\end{tabular}




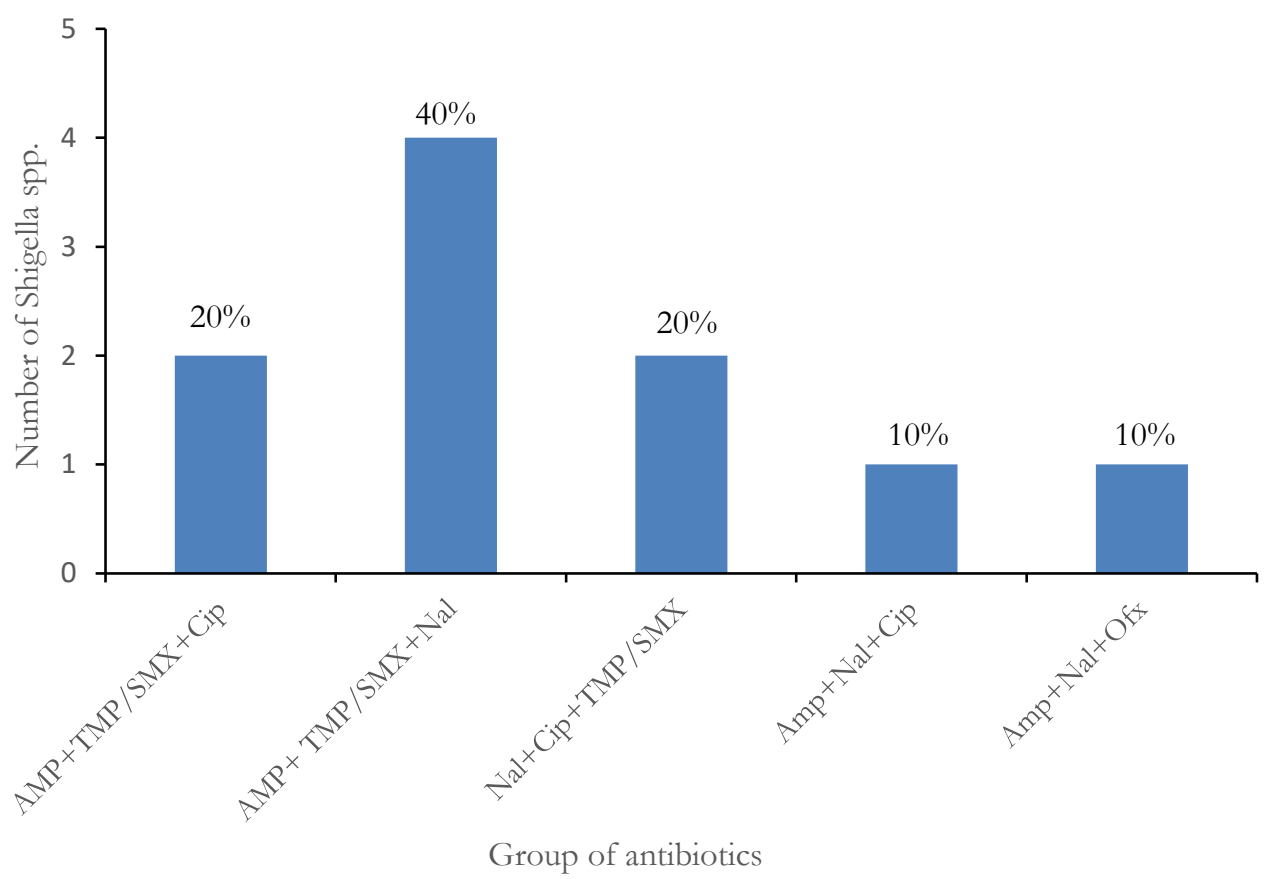

Figure 2. Bar-graph showing multi-drug resistance (MDR) pattern of Shigella species. (Amp-Ampicillin, TMP/SMXCotrimoxazole, Cip- Ciprofloxacin, Nal- Nalidixic acid, Ofx-Ofloxacin).

\section{DISCUSSION}

This study was conducted from April to August 2014 among the patients suspected of acute gastroenteritis visiting Sukraraj Tropical and Infectious Disease Hospital, Teku. Out of 653 studied cases, 25(3.82\%) cases were Shigella positive. This isolation rate is comparable to a study done in Tribhuvan University Teaching Hospital and Kanti Hospital (2.1\% culture positive) (Dhital et al., 2017). In contrast to our findings, two different studies conducted in Nepal reported a relatively higher prevalence rate $(13.61 \%$ and $10.8 \%)$ (Wilson et al., 2006; Khan et al., 2014). The prevalence of food and water-borne infections like Shigellosis increases in the rainy season as the infection rate fluctuates according to the seasons. Our study shows that the patients in the age group 16-45 years were more susceptible to Shigella infection $(16(64 \%))$. However, there was no significant difference in the infection rate among all the age groups. In contrast to our study, the infection rate was higher in the age group $<10$ years in most studies (Kansakar et al., 2006; Wilson et al., 2006; Khan et al., 2014; Mengistu et al., 2014). This discrepancy is because the children usually visit the children's hospitals in Kathmandu rather than the tertiary care hospital where this study was carried out. This is also reflected in our study, as participants below the age of 10 were lower than other age groups.

S. flexneri $[18(72 \%)]$ was found to be the predominant Shigella species in our study, followed by $S$. dysenteriae [6(28\%)]. This finding is congruent to hospitalbased studies in Nepal (Wilson et al., 2006; Khan et al., 2014), whereas incongruent to a study done in eastern Nepal where the predominant isolate was $S$. dysenteriae $(73.6 \%)$ followed by $S$. flexneri $(23 \%)$ and $S$. boydii (4\%) (Bhattacharya et al., 2005). In the studies in Nepal from 1995 to 2002, the prevalence of $S$. dysenteriae was higher than the $S$. flexneri, while in the studies done from 2003 to 2005, S. flexneri was found to be the predominant species (Kansakar et al., 2006). The recent studies conducted in India (Puzari et al., 2018), Nepal (Maharjan et al., 2017; Thapa et al., 2017), Iran (Nikfar et al., 2017), China (Chang et al., 2016), and Latin America (Sati et al., 2019) show that S. flexneri is the predominant species. On the other hand, nations such as England (Bardsley et al., 2020), Australia (O’Sullivan et al., 2002; Ingle et al., 2020), and the United States (Kozyreva et al., 2016) showed a higher predominance of $S$. sonnei. These findings support the claim that $S$. flexneri infection is associated with developing and underdeveloped countries, and $S$. sonnei infection is associated with developed countries (Kotloff et al., 2018; Bardsley et al., 2020). However, a rapid rise in the $S$. sonnei cases has been observed in developing countries in recent years (Anderson et al., 2016). This could be possibly associated with the climatic and environmental changes and the innate changing nature of Shigella serogroups and serotypes from time to time. 
All the antibiotics used in our study were the routine antibiotics used in the diagnostic microbiology laboratory in Sukraraj Tropical and Infectious Disease Hospital, Teku, Kathmandu. Our study showed the increased resistance to Nalidixic acid (80\%), Ampicillin (72\%), Cotrimoxazole (52\%), and Ciprofloxacin (36\%) corresponding to various studies conducted in different parts of the world (Srinivasa et al., 2009; Bhattacharya et al., 2012; Gharibi et al., 2012). Although further confirmation is needed, our study suggests that Nalidixic acid, Cotrimoxazole, Ampicillin, and Ciprofloxacin can be used no longer for the empirical treatment of shigellosis. Similarly, Nalidixic acid is a drug of choice in developing countries, and Ciprofloxacin in developed countries (Gharibi et al., 2012). The increased resistance ( $80 \%$ resistance) of Nalidixic acid was observed in the present study and other studies conducted in Nepal (Bhattacharya et al., 2005; Khan et al., 2014; Dhital et al., 2017). This increased resistance to Nalidixic acid in Nepal can be attributed to the over-the-counter availability of drugs, self-prescription by patients, and poor antibiotic policies. In the early 1990s, Shigella isolates were susceptible to most prescribed antibiotics such as Gentamicin, Norfloxacin, and Nalidixic acid (Jesudason, 2002; Dutta et al., 2003; Rahbar et al., 2007). However, during the late 1990s, Shigella isolates were resistant to most of these antibiotics but susceptible to Ciprofloxacin (Sack et al., 1997). A study conducted in Bangladesh has shown the rising trend of Ciprofloxacin resistance, i.e., from 0\% in 2004 to 48\% in 2010 (Azmi et al., 2014). A similar study of the Shigella isolates between 2010 and 2015 revealed South-Asia as a reservoir for the global spread of Ciprofloxacin resistant S. sonnei (Chung The et al., 2016). Similarly, a study conducted from the Shigella isolates over the six years from 2011 to 2016 in Iran also suggested the higher resistance $(76 \%)$ to Ciprofloxacin (Mamishi et al., 2019). These statistics show that the Ciprofloxacin-resistant Shigella species has increased considerably over time. Although WHO has recommended Ciprofloxacin as a drug of choice to treat shigellosis (WHO, 2005), our result suggests that Ciprofloxacin cannot be trusted blindly as the effective drug against shigellosis.

According to our findings, cefotaxime, a third-generation cephalosporin, was the most sensitive (100\% of Shigella isolates were susceptible to it). This result is congruent to the studies performed by Dhital et al. (2017), where $93 \%$ of Shigella isolates were susceptible to Ceftriaxone, and by Kansakar et al. (2006), where 100\% of Shigella isolates were susceptible. In similar settings, Maharjan et al. (2017) found $97 \%$ of Shigella species, and Thapa et al. (2017) found $71.4 \%$ of Shigella species susceptible to Ceftriaxone. Several studies across the world have also shown the increased resistance of Shigella species to third-generation cephalosporins (Radice et al., 2001; Vinh et al., 2009; Mandal et al., 2010). More recent research also consistently supports the fact that third- generation cephalosporins cannot be relied on treating Ciprofloxacin resistant Shigella species due to surge in third-generation cephalosporin-resistant Shigella species (Hosseini Nave et al., 2016; Nüesch-Inderbinen et al., 2016; Madhavan et al. 2018; Abbasi et al., 2019; Bian et al., 2019). Therefore, although our study has shown the high susceptibility of Shigella species to a third-generation cephalosporin (Cefotaxime), we still suggest using thirdgeneration cephalosporin with caution. Our study was conducted seven years ago, and recent studies across the world have shown increased resistance of Shigella species to third-generation cephalosporins. So, for further studies, we recommend carrying out the study in similar settings with few other third-generation cephalosporins included before concluding with certainty that thirdgeneration cephalosporins are the effective options for treating shigellosis.

Forty percent (40\%) of the Shigella-positive isolates were MDR. This figure corresponds to a study conducted in the hospital settings in Pokhara (74.7\%) and Kathmandu (86\%) (Wilson et al., 2006; Dhital et al., 2017). MDR isolates in our study were resistant to Nalidixic acid, Ciprofloxacin, Cotrimoxazole, and Ampicillin, and Ofloxacin. The emergence of MDR Shigella isolates is of great concern in South Asian countries, including Nepal (Rahman et al., 2004). The high resistance level in Shigella can be attributed to the transfer of resistant determinants such as plasmids, integrons and transposons (Barrantes \& Achí, 2016). Due to the rise in resistance to thirdgeneration cephalosporins (Abbasi et al., 2019), a recent study has suggested using Azithromycin and Piperacillin tazobactam as an alternative (Madhavan et al., 2018). However, resistance to Azithromycin has also been reported (Nüesch-Inderbinen et al., 2016). Therefore, we can see that the MDR Shigella species is increasing continuously. Continuous monitoring of the MDR strains and an effective antibiotic prescription policy in Nepal could help mitigate increased drug resistance seen in Shigella species.

\section{CONCLUSIONS}

Our study found the predominant Shigella serovar to be Shigella flexneri. Shigella species were highly resistant to commonly prescribed antibiotics in Nepal, such as Nalidixic acid, Ampicillin, Cotrimoxazole, and Ciprofloxacin, whereas susceptible to a third-generation cephalosporin (Cefotaxime). We found a higher prevalence of MDR Shigella, and we suggest using thirdgeneration cephalosporins to treat MDR Shigella infection.

\section{ACKNOWLEDGEMENTS}

We extend our sincere gratitude to all the staff in Microbiology Department in Sukraraj Tropical and Infectious disease Hospital, Teku, Kathmandu. Besides, we highly appreciate all the suggestions and guidance 
provided by the teaching staff in the Department of Microbiology, Kirtipur.

\section{AUTHOR CONTRIBUTIONS}

$\mathrm{BB}$ contributed to the study design, laboratory work, and manuscript writing. DN performed data analysis and manuscript writing. JA and SB contributed to the conceptualization and study design.

\section{CONFLICT OF INTEREST}

The authors declare no competing interest.

\section{DATA AVAILABILITY STATEMENT}

The data that support the findings of this study are available from the corresponding author, upon reasonable request.

\section{REFERENCES}

Abbasi, E., Abtahi, H., van Belkum, A., \& Ghaznavi-Rad, E. (2019). Multidrug-resistant Sbigella infection in pediatric patients with diarrhea from central Iran. Infection and Drug Resistance, 12, 1535-1544. https://doi.org/10.2147/IDR.S 203654.

Anderson, M., Sansonetti, P.J., \& Marteyn, B.S. (2016). Shigella diversity and changing landscape: Insights for the twentyfirst century. Frontiers in Cellular and Infection Microbiology, 6, 45. https://doi.org/10.3389/fcimb.2016.00045.

Arnold, S.L.M. (2021). Target product profile and development path for Shigellosis treatment with antibacterials. ACS Infectious Diseases, 7(5), 948-958. https://doi.org/10.1021/a csinfecdis.0c00889.

Azmi, I.J., Khajanchi, B.K., Akter, F., Hasan, T.N., Shahnaij, M., Akter, M., Banik, A., Sultana, H., Hossain, M.A., Ahmed, M.K., Faruque, S.M., \& Talukder, K.A. (2014). Fluoroquinolone resistance mechanisms of Shigella flexneri isolated in Bangladesh. PloS One, 9(7), e102533.

Bardsley, M., Jenkins, C., Mitchell, H.D., Mikhail, A.F.W., Baker, K.S., Foster, K., Hghes, G., \& Dallman, T.J. (2020). Persistent transmission of shigellosis in England is associated with a recently emerged multidrug-resistant strain of Shigella sonnei. Journal of Clinical Microbiology, 58(4), e01692. https://doi.org/10.1128/JCM.01692-19.

Barrantes, K., \& Achí, R. (2016). The importance of integrons for development and propagation of resistance in Shigella: the case of Latin America. Brazilian Journal of Microbiology, 47, 800-806.

Bhattacharya, D., Sugunan, A.P., Bhattacharjee, H., Thamizhmani, R., Sayi, D.S., Thanasekaran, K., Manimunda, S.P., Ghosh, A.R., Bharadwaj, A.P., Singhania, M., \& Roy, S. (2012). Antimicrobial resistance in Sbigellarapid increase \& widening of spectrum in Andaman Islands, India. The Indian Journal of Medical Research, 135(3), 365.

Bhattacharya, S., Khanal, B., Bhattarai, N.R., \& Das, M.L. (2005). Prevalence of Shigella species and their antimicrobial resistance patterns in Eastern Nepal. Journal of Health, Population and Nutrition, 23(4), 339-342.

Bian, F., Yao, M., Fu, H., Yuan, G., Wu, S., \& Sun, Y. (2019). Resistance characteristics of CTX-M type Shigella flexneri in China. Bioscience Reports, 39(9), BSR20191741. https://doi .org/10.1042/BSR20191741.

Chang, Z., Zhang, J., Ran, L., Sun, J., Liu, F., Luo, L., Zeng, L., Wang, L., Li, Z., Yu, H., \& Liao, Q. (2016). The changing epidemiology of bacillary dysentery and characteristics of antimicrobial resistance of Shigella isolated in China from 2004-2014. BMC Infectious Diseases, 16(1), 685. https://doi. org/10.1186/s12879-016-1977-1.

Chung The, H., Rabaa, M.A., Pham Thanh, D., De Lappe, N., Cormican, M., Valcanis, M., Howden, B.P., Wangchuk, S., Bodhidatta, L., Mason, C.J., Nguyen Thi Nguyen, T., Vu Thuy, D., Thompson, C.N., Phu Huong Lan, N., Voong Vinh, P., Ha Thanh, T., Turner, P., Sar, P., Thwaites, G., Thomson, N.R., Holt, K.E., \& Baker, S. (2016). South Asia as a reservoir for the global spread of Ciprofloxacinresistant Shigella sonnei: A cross-sectional study. PLoS Medicine, 13(8), 1-12. https://doi.org/10.1371/journal.pm ed.1002055.

CLSI. (2014). Clinical and Laboratory Standards Institute. Performance Standards for Antimicrobial Susceptibility Testing; Twenty-Fourth Informational Supplement. CLSI Wayne, PA.

Dhital, S., Sherchand, J.B., Pokharel, B.M., Parajuli, K., Mishra, S.K., Sharma, S., Kattel, H.P., Khadka, S., Khatiwada, S., \& Rijal, B. (2017). Antimicrobial susceptibility pattern of Shigella spp. isolated from children under 5 years of age attending tertiary care hospitals, Nepal along with first finding of ESBL-production. BMC Research Notes, 10(1), 192. https://doi.org/10.1186/s13104-017-2512-1.

Dutta, S., Dutta, D., Dutta, P., Matsushita, S., Bhattacharya, S. K., \& Yoshida, S. (2003). Shigella dysenteriae serotype 1, Kolkata, India. Emerging Infectious Diseases, 9(11), 1471-1474.

Gharibi, O., Zangene, S., Mohammadi, N., Mirzaei, K., Karimi, A., Gharibi, A., \& Khajehiean, A. (2012). Increasing antimicrobial resistance among Sbigella isolates in the Bushehr, Iran. Pakistan Journal of Biological Sciences, 15(3), 156159. https://doi.org/10.3923/pjbs.2012.156.159.

Heymann, D. L. (2008). Control of communicable diseases manual. American Public Health Association.

Hosseini Nave, H., Mansouri, S., Sadeghi, A., \& Moradi, M., (2016). Molecular diagnosis and anti-microbial resistance patterns among Shigella spp. isolated from patients with diarrhea. Gastroenterology and Hepatology from Bed to Bench, 9(3), 205-210. https://doi.org/10.22037/ghfbb.v0 i0.809.

Ingle, D.J., Andersson, P., Valcanis, M., Barnden, J., da Silva, A. G., Horan, K.A., Seemann, T., Easton, M., Williamson, D. A., Sherry, N.L., \& Howden, B.P. (2020). Prolonged outbreak of multidrug-resistant Shigella sonnei Harboring bla (CTX-M-27) in Victoria, Australia. Antimicrobial Agents and Chemotherapy, 64(12), e01518-20. https://doi.org/10.1128/ AAC.01518-20.

Jesudason, M.V. (2002). Shigella isolation in Vellore, south India (1997-2001). Indian Journal of Medical Research, 115, 11-13.

Kansakar, P., Malla, S., \& Ghimire, G.R. (2006). Shigella isolates of Nepal: Changes in the incidence of Shigella subgroups and trends of antimicrobial susceptibility pattern. Kathmandu University Medical Journal, 5(17), 32-37.

Khan, S., Singh, P., Ansari, M., \& Asthana, A. (2014). Isolation of Shigella species and their resistance patterns to a panel of fifteen antibiotics in mid and far western region of Nepal. Asian Pacific Journal of Tropical Disease, 4(1), 30-34. https://doi.org/10.1016/S2222-1808(14)60309-1.

Khan, S., Singh, P., Asthana, A., \& Ansari, M. (2013). Magnitude of drug resistant shigellosis in Nepalese patients. Iranian Journal of Microbiology, 5(4), 334-338.

Kotloff, K.L., Riddle, M.S., Platts-Mills, J.A., Pavlinac, P., \& Zaidi, A.K.M. (2018). Shigellosis. The Lancet, 391(10122), 801-812. https://doi.org/10.1016/S0140-6736(17)332968.

Kozyreva, V.K., Jospin, G., Greninger, A.L., Watt, J.P., Eisen, 
J.A., \& Chaturvedi, V. (2016). Recent outbreaks of shigellosis in California caused by two distinct populations of Shigella sonnei with increased virulence or fluoroquinolone resistance. $m S$ phere, 1(6), e00344-16. https://doi.org/10.11 28/msphere.00344-16.

Lima, A.A., Lima, N.L., Pinho, M.C., Barros, E.A., Teixeira, M. J., Martins, M.C., \& Guerrant, R.L. (1995). High frequency of strains multiply resistant to ampicillin, trimethoprimsulfamethoxazole, streptomycin, chloramphenicol, and tetracycline isolated from patients with shigellosis in northeastern Brazil during the period 1988 to 1993. Antimicrobial Agents and Chemotherapy, 39(1), 256-259.

Madhavan, A., Balakrishnan, S., \& Vasudevapanicker, J. (2018). Antibiotic susceptibility pattern of Shigella isolates in a tertiary healthcare center. Journal of Laboratory Physicians, 10(02), 140-144. https://doi.org/10.4103/ilp.jlp_93_17.

Maharjan, S., Rayamajhee, B., Shreshtha, A., \& Acharya, J. (2017). Serotyping and antibiotic susceptibility patterns of Vibrio and Shigella isolates from diarrheal patients visiting a Tropical and Infectious Diseases Hospital in central Nepal. BMC Research Notes, 10, 626. https://doi.org/10.1186/s13 104-017-2967-0.

Mamishi, S., Arab Yazdi, Z., Mahmoudi, S., Moradzadeh, M., Taghi Haghi Ashtiani, M., \& Pourakbari, B. (2019). Antimicrobial-resistance pattern of Shigella species in children: a six-year study in an Iranian referral Hospital. Ann Ig, 31(4), 356-364.

Mandal, J., Mondal, N., Mahadevan, S., \& Parija, S.C. (2010). Emergence of resistance to third-generation cephalosporin in Shigella - a case report. Journal of Tropical Pediatrics, 56(4), 278-279.

McCartney, J.E., Collee, J.G., \& Mackie, T.J. (1989). Practical medical microbiology. Charchil Livingstone.

Mengistu, G., Mulugeta, G., Lema, T., \& Aseffa, A. (2014). Prevalence and antimicrobial susceptibility patterns of Salmonella serovars and Shigella species. Journal of Microbial \& Biochemical Technology, 6(S2), https://doi.org/10.4172/19 48-5948.S2-006.

Nikfar, R., Shamsizadeh, A., Darbor, M., Khaghani, S., \& Moghaddam, M. (2017). A study of prevalence of Shigella species and antimicrobial resistance patterns in paediatric medical center, Ahvaz, Iran. Iranian Journal of Microbiology, 9(5), 277-283.

Nüesch-Inderbinen, M., Heini, N., Zurfluh, K., Althaus, D., Hächler, H., \& Stephan, R. (2016). Shigella antimicrobial drug resistance mechanisms, 2004-2014. Emerging Infectious Diseases, 22(6), 1083-1085. https://doi.org/10.3201/eid22 06.152088 .

O'Sullivan, B., Delpech, V., Pontivivo, G., Karagiannis, T., Marriott, D., Harkness, J., \& McAnulty, J.M. (2002). Shigellosis linked to sex venues, Australia. Emerging Infectious Diseases, 8(8), 862-864. https://doi.org/10.3201/eid0808 .010534 .

Puzari, M., Sharma, M., \& Chetia, P. (2018). Emergence of antibiotic resistant Shigella species: A matter of concern. Journal of Infection and Public Health, 11(4), 451-454. https://doi.org/10.1016/j.jiph.2017.09.025.

Radice, M., Gonzealez, C., Power, P., Vidal, M., \& Gutkind, G. (2001). Third-generation cephalosporin resistance in Shigella sonnei, Argentina. Emerging Infectious Diseases, 7(3), 442-443. https://doi.org/10.3201/eid0703.010313.

Rahbar, M., Deldari, M., \& Hajia, M. (2007). Changing prevalence and antibiotic susceptibility patterns of different Shigella species in Tehran, Iran. The Internet Journal of Microbiology, 3(2), 1-4.

Rahman, M., Shoma, S., Rashid, H., Siddique, A.K., Nair, G.B., \& Sack, D.A. (2004). Extended-spectrum $\beta$-lactamasemediated third-generation cephalosporin resistance in Shigella isolates in Bangladesh. Journal of Antimicrobial Chemotherapy, 54(4), 846-847.

Sack, R.B., Rahman, M., Yunus, M., \& Khan, E.H. (1997). Antimicrobial resistance in organisms causing diarrheal disease. Clinical Infectious Diseases, 24(Suppl 1), S102-S105. https://doi.org/10.1093/clinids/24.supplement_1.s102.

Sati, H.F., Bruinsma, N., Galas, M., Hsieh, J., Sanhueza, A., Ramon Pardo, P., \& Espinal, M.A. (2019). Characterizing Shigella species distribution and antimicrobial susceptibility to ciprofloxacin and nalidixic acid in Latin America between 2000-2015. PloS One, 14(8), e0220445-e0220445. https://doi.org/10.1371/journal.pone.0220445.

Srinivasa, H., Baijayanti, M., \& Raksha, Y. (2009). Magnitude of drug resistant Shigellosis: a report from Bangalore. Indian Journal of Medical Microbiology, 27(4), 358-360.

Taneja, N., Mewara, A., Kumar, A., Verma, G., \& Sharma, M. (2012). Cephalosporin-resistant Shigella flexneri over 9 years (2001-09) in India. Journal of Antimicrobial Chemotherapy, 67(6), 1347-1353.

Thapa, K., Bhattachan, B., Gurung, R.R., Katuwal, A., \& Khadka, J.B. (2017). Antibiotic resistance pattern of Shigella spp. among gastroenteritis patients at Tertiary Care Hospital in Pokhara, Nepal. Nepal Journal of Biotechnology, 5(1), 14-20. https://doi.org/10.3126/njb.v5i1.18865.

Vinh, H., Baker, S., Campbell, J., Hoang, N.V.M., Loan, H.T., Chinh, M.T., Ahn, V.T.C., Diep, T.S., Phuong, L.T., Schultsz, C., \& Farrar, J. (2009). Rapid emergence of third generation cephalosporin resistant Shigella spp. in southern Vietnam. Journal of Medical Microbiology, 58(2), 281-283.

von Seidlein, L., Kim, D.R., Ali, M., Lee, H., Wang, X., Thiem, V.D., Canh, D.G., Chaicumpa, W., Agtini, M.D., Hossain, A., Bhutta, Z.A., MAson, C., Sethabutr, O., Talukder, K., Nair, G.B., Deen, J.L., Kotloff, K., \& Clemens, J. (2006). A multicentre study of Sbigella diarrhoea in six Asian countries: disease burden, clinical manifestations, and microbiology. PLoS Medicine, 3(9), e353. https://doi.org/10 .1371 /journal.pmed.0030353.

WHO. (2005). Guidelines for the control of shigellosis, including epidemics due to Shigella dysenteriae type 1. World Health Organization.

Wilson, G., Easow, J.M., Mukhopadhyay, C., \& Shivananda, P. G. (2006). Isolation \& antimicrobial susceptibility of Shigella from patients with acute gastroenteritis in western Nepal. Indian Journal of Medical Research, 123(2), 145-150. 